\title{
High Resolution Optical and Vibrational Spectroscopy with Low Loss EELS
}

\author{
Paul Cueva, ${ }^{1}$ and David A. Muller ${ }^{1,2}$ \\ ${ }^{1}$ School of Applied and Engineering Physics, Cornell University, Ithaca, NY 14853 \\ ${ }^{2}$ Kavli Institute at Cornell for Nanoscale Science, Ithaca, NY 14853
}

Despite being diffraction limited to thousands of Ångstroms, infrared and optical spectroscopies remain effective characterization techniques. While it is possible to perform subatomic resolution electron energy loss spectroscopy (EELS) at unprecedented energy resolution, with ever improving aberration correctors and monochromators, it is argued that low loss features can be severely delocalized (up to hundreds of Ångstroms for optical excitations and millions of Ångstroms for vibrational states) which would suggest poor prospects for atomic resolution imaging [1]. Here, we go beyond the traditional multipole expansions and construct an exact treatment of the near-field inelastic scattering that predicts atomic-scale resolution for monopolar optical and vibrational excitations.

While the dipole approximation succeeds for high loss, large probe experiments, very-low loss experiments enter a regime where multipole expansions converge poorly and no longer hold [2,3]. By removing the multipole approximation and implementing the exact transition potential, we show that it is possible to image surprisingly low loss transitions at the Ångstrom scale. Interestingly, we have found that the monopole term is scale invariant and therefore contributes a high-resolution component even in the low loss limit. By treating the incident electron beam as a point source as opposed to a plane wave, we can solve the perturbation analytically to obtain a Green's function, which can then be convolved with a known probe shape to approximate the full spatially resolved inelastic scattering probability. The work we present investigates transitions of a solvable hydrogenic atom and harmonic vibrational mode, but the selection rules and qualitative behavior remain the same for any system.

We predict a FWHM of $1.5 \AA$, for a $1 \mathrm{eV}$ hydrogenic optical transition induced by a $100 \mathrm{keV}$ beam (Fig. 1) due to large monopole contribution. The approach also works for non-hydrogenic excitations such as vibrational modes. For a $100 \mathrm{meV}$ phonon in the harmonic approximation with bond length fluctuation of $0.1 \AA$, we calculate a resolution the object function of is $\sim 0.5 \AA$ (five times the wavefunction size). Furthermore, in this near field regime, we have a new set of selection rules. Angular momentum conservation prohibits on-atom transitions between states with $\Delta \mathrm{m} \neq 0$. Furthermore, donut shaped profiles are seen for $\Delta \mathrm{m}= \pm 1$, in the absence of elastic scattering, which can wash the profiles out. These results predict that the new generation of corrected, monochromated microscopes are able to resolve low loss (optical/infrared) transitions and may eventually be able to probe vibrational spectra at the atomic scale. Such characterization would allow for better understanding of behavior at the molecular scale [5].

\section{References}

[1]R. F. Egerton, J. Elec. Microsc., 48(6) (1999) 711-716

[2] J. M. Auerhammer and P. Rez, Phys. Rev. B, 40 (1989) 2024-2030

[3] D. W. Essex, et al., Ultramicroscopy, 80(3) (1999) 183-192

[4] R. F. Egerton, EELS in the Electron Microscope, equation 5.17

[5] Facilities support from the Cornell Center for Materials Research, an NSF MRSEC (DMR1120296). PC acknowledges support from the NSF IGERT program (DGE-0903653). 

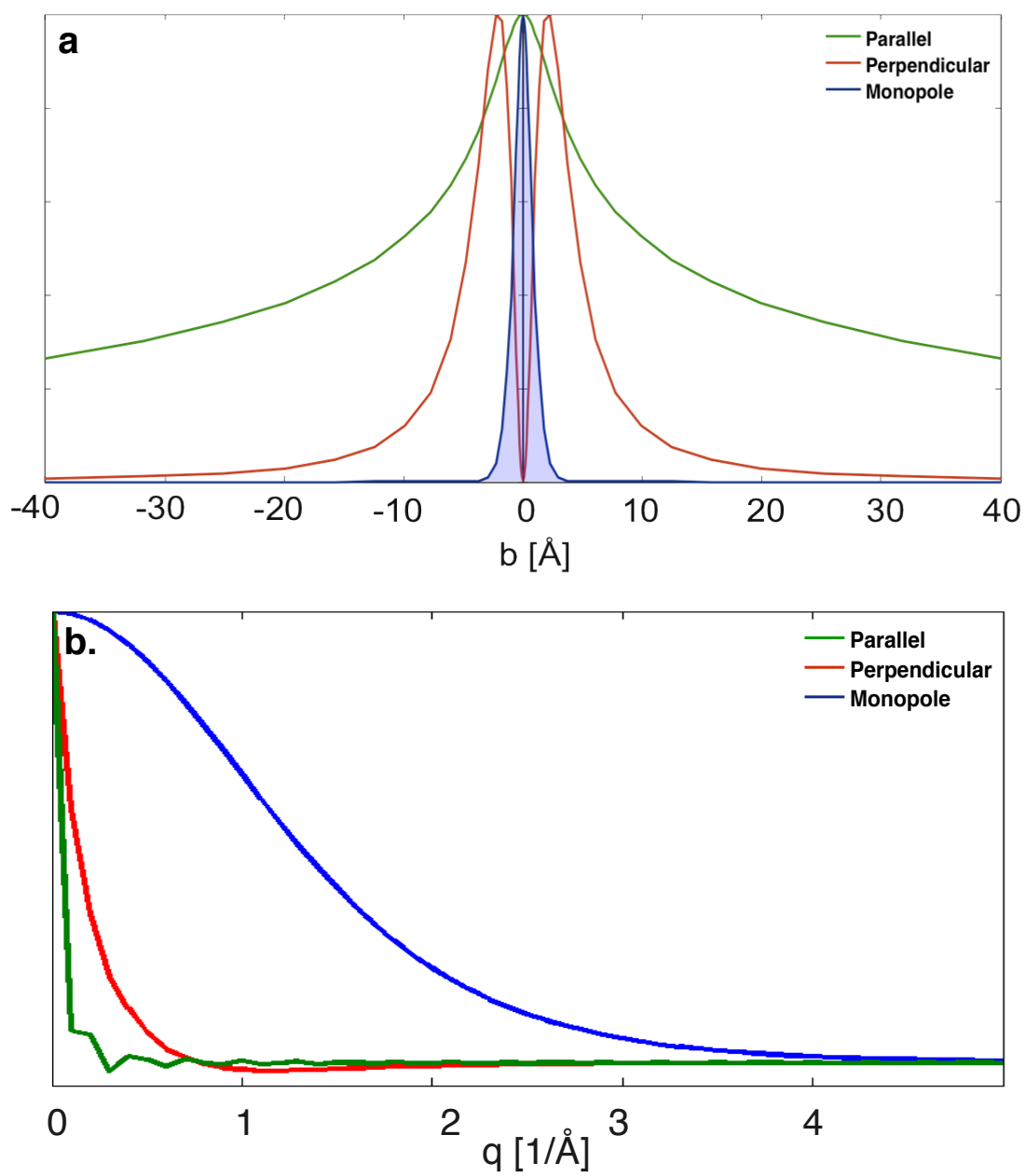

Figure 1. a) Normalized transition probabilities for the monopole and two dipole terms of a leV hydrogenic transition vs impact parameter $\mathrm{b}$. The dipole transitions are delocalized while the monopole remains tightly bound. Notably, the tails of the distributions extend well beyond the size of the full-width half max, implying that a large amount of the scattering will occur far away from the atom. b) he corresponding contrast transfer function indicates monopole contrast out to an angstrom

Figure 2. (a) Full-width half-max and (b) R.80\% of hydrogenic transition for varying values in energy loss. The monopole transition remains near the size of the wave function, while the dipole transitions can be orders of magnitude larger. The dipole theory[4] predicts a dependency of $\mathrm{FWHM} \sim \mathrm{E}^{3 / 4}$ 